\title{
106 THE EFFECTS OF ENDOSCOPIC SEVERITY AND BIOLOGIC TREATMENT ON LEVELS OF DEPRESSION AND DISABILITY AMONGST IBD PATIENTS:A CASE CONTROL STUDY
}

M Khan, P Calpin, O Kelly, J Moloney, E McDermott, H Mulcahy, A Aftab, G Courtney Gastroenterology, St Luke's Hospital Kilkenny (2) Gastroenterology, St Vincent's University Hospital, Dublin 4, Ireland

\subsection{6/gutjnl-2013-305143.106}

Introduction As with other chronic diseases, patients with IBD are also at risk for depression, anxiety, low self-esteem and social isolation. The physical symptoms in gastrointestinal disease, including diarrhoea, bleeding per rectum and faecal incontinence are much less socially acceptable than other chronic diseases. Together with the potential for surgery, including stoma formation, this results in a high risk for altered body image and psychosocial distress unique to IBD.

Aims/Background In this study we aimed to compare the effects of disease activity on body image and quality of life in these patients and also to assess whether biological treatment offers superior clinical and psychological outcomes or reduction in disability.

Method Patients taking biologics attending St. Luke's IBD service were asked to complete a questionnaire including the Body Image States Scale (BISS), the Stunkard Figure Rating Scales (FRS), the Hospital Anxiety and Depression scale (HADS), the Beck Depression Inventory (BDI), the SF 36 Health Survey, the IBDQ and the sexual satisfaction survey. Clinical markers of disease severity were also examined including recent endoscopic findings, Mayo score and CRP. These were then age and sex matched with IBD patients not taking biologics. Results were statistically analysed $\mathrm{p}$ values $<0.05$ were deemed significant.

Results 64 IBD patients were included in this study. 32 patients were taking biologics at time of study $(60 \% \mathrm{M})$ Median age was 
41; IQR (31-54). 55\% had UC, 33\% had Crohn's, 13\% had indeterminate colitis. These were age, sex and disease matched with 32 respondents not taking biologics. Mayo scores, $\mathrm{Hb}$ and CRP were not significantly different between groups. Endoscopic severity was increased in the biologic group though not significantly. There was no significant difference in Beck Depression Inventory scores between treatment groups, however those on biologic treatment who were in remission reported significantly lower disability using the Disability Index (Peyronie et al, 2012) compared to their peers in remission not on biologics $(p<0.05)$. Endoscopic severity was found to be independently associated with higher Beck depression Inventory scores $(\mathrm{p}<0.01)$.

Conclusion Depression is common in IBD. Clinical and mucosal remission may not only lead to reduced inflammation but also to reduced levels of depression. Treatment with biologics appears to be associated with lower levels of disability in patients with similar pre-treatment clinical severity scores. 